Article

\title{
Genetic Diversity of Bovine Hemoprotozoa in South Korea
}

\author{
Dongmi Kwak ${ }^{1,2}$ and Min-Goo Seo ${ }^{3, *(1)}$ \\ College of Veterinary Medicine, Kyungpook National University, Daegu 41566, Korea; dmkwak@knu.ac.kr \\ Cardiovascular Research Institute, Kyungpook National University, Daegu 41944, Korea \\ 3 Veterinary Drugs and Biologics Division, Animal and Plant Quarantine Agency, Gyeongbuk 39660, Korea \\ * Correspondence: koreasmg@korea.kr
}

Received: 21 August 2020; Accepted: 19 September 2020; Published: 20 September 2020

\begin{abstract}
Tick-borne pathogens cause economically significant diseases in cattle. Theileria spp. are parasitic protozoa and the causative agent of bovine theileriosis. Here we report the distribution and risk factors of bovine Theileria using blood samples taken between 2018 and 2019. Of 737 tested cattle, nine animals $(1.2 \%)$ were positive for Theileria orientalis infection by $18 \mathrm{~S}$ rRNA gene amplification. Further analysis of the infected samples using the T. orientalis major piroplasm surface protein (MPSP) gene revealed five different genotypes circulating in the population: Types 1, 2, 3, 7, and N3. To the best of our knowledge, this is the first research to describe the existence of the T. orientalis MPSP genotype N3 in South Korea. Although the prevalence of bovine T. orientalis was low, our study offers data on the geographical distribution and prevalence of bovine Theileria spp. in South Korea. Further studies are warranted to determine the correlation of clinical symptoms with parasite MPSP genotypes. Our data provide epidemiological information to help control bovine theileriosis in South Korea.
\end{abstract}

Keywords: cattle; piroplasm; phylogeny; Theileria

\section{Introduction}

Tick-borne pathogens (TBPs) cause significant morbidity and mortality in mammals, representing a major public health threat and damaging livestock production [1]. The piroplasms Babesia and Theileria are widely distributed intracellular parasitic protozoans that infect erythrocytes, or erythrocytes and lymphocytes, respectively, and are economically significant TBPs of domestic and wild animals [2].

Theileria spp. (Apicomplexa: Piroplasmida: Theileriidae) are transmitted by ticks and cause bovine theileriosis, an economically important disease in cattle [3]. Infected animals display a range of symptoms, including chronic anemia, fever, reduced milk production, weight loss, anorexia, and jaundice [4]. Several species of Theileria infect ruminants, with a wide variation in pathogenicity. Theileria parva and T. annulata are known to cause East Coast fever and tropical theileriosis, respectively. Both species are highly pathogenic in cattle. A third species, T. orientalis, was previously believed to induce only mild or asymptomatic disease [5]; however, it has recently emerged as a virulent parasite that can cause clinical theileriosis and has resulted in notable economic impact to the Asia-Pacific cattle industry [6]. These species, including T. buffeli, T. orientalis, and T. sergenti, are considerably similar, and the discrete taxonomy of this group is debatable. On the basis of molecular approaches, the three species of parasites are currently categorized as one species, namely T. orientalis; however, a study has proposed that it should be called the T. orientalis complex [4]. T. orientalis belongs to a comparatively benign group of Theileria with universal existence. It causes little pathogenicity in bovine compared with T. parva and T. annulata; however, it has caused considerable economic loss in 
China [7], South Korea [8-11], Japan [12], Australia [6], and Vietnam [13] due to decreased production of meat, milk, and other by-products.

The active areas and prevalence of ticks are important factors in the epidemiology of Theileria species in cattle [7]. In South Korea, Haemaphysalis longicornis, which is the most prevalent tick species, is a primary vector of T. orientalis $[14,15]$. T. orientalis has been detected in H. longicornis infesting cattle in South Korea [15]. Although T. orientalis causes low virulence in healthy cattle, in endemic areas, older cattle with subclinical theileriosis may remain long-term carriers of piroplasm and may act as a reservoir of infection for tick and other animals $[5,8]$. Here, we investigated the distribution and risk factors of bovine T. orientalis in South Korea and collected epidemiological information needed to control piroplasm infections in South Korea.

\section{Results}

\section{1. $P C R$ and Molecular Identification}

We used the $18 \mathrm{~S}$ rRNA sequences of $T$. orientalis to detect infections in cattle $(9 / 737,1.2 \% ; 95 \%$ confidence interval (CI): 0.4-2.0). Additional genetic analysis of the positive samples revealed that the cattle were also positive for the T. orientalis major piroplasm surface protein (MPSP) gene (9/737, 1.2\%; 95\% CI: 0.4-2.0) (Table 1). The prevalence of cattle positive for T. orientalis varied for each group (Table 1). Female cattle $(p=0.0293)$ had a greater possibility to be positive for T. orientalis than male cattle. In addition, cattle over 4 years old $(p=0.0176)$ had a greater possibility to be positive for T. orientalis than other age groups. Moreover, we detected differences in breed and region susceptibilities. Only the Holstein breed $(p=0.0079)$ tested positive for T. orientalis, with no infections detected in the native Korean brown cattle, Hanwoo breed. On the other hand, the Gyeongnam Province cattle $(p=0.0451)$ were positive for $T$. orientalis, with no infections detected in cattle from the Gyeongbuk Province. Furthermore, only samples taken in summer $(p=0.0166)$ were positive for T. orientalis compared with those in other seasons. Babesia spp. was not detected in this study.

Table 1. Distribution of bovine Theileria orientalis in South Korea in 2018-2019.

\begin{tabular}{|c|c|c|c|c|c|c|}
\hline \multicolumn{2}{|c|}{ Category } & No. Tested & No. Positive (\%) & $\mathbf{R R}^{1}$ & $95 \% \mathrm{CI}^{2}$ & $p$-Value \\
\hline \multirow{2}{*}{ Sex } & Female & 327 & $8(2.4)$ & 10.0 & $1.3-79.8$ & 0.0293 * \\
\hline & Male & 410 & $1(0.2)$ & 0.1 & $0.01-0.8$ & 0.0293 * \\
\hline \multirow{3}{*}{ Age } & $<2$ & 122 & 0 & 0.3 & $0.02-4.5$ & 0.357 \\
\hline & $2-4$ & 413 & $3(0.7)$ & 0.4 & $0.1-1.6$ & 0.1832 \\
\hline & $>4$ & 202 & $6(3.0)$ & 5.3 & $1.3-21.0$ & $0.0176^{*}$ \\
\hline \multirow{2}{*}{ Breed } & Hanwoo & 525 & 0 & 0.02 & $0-0.9$ & 0.0079 * \\
\hline & Holstein & 212 & $9(4.2)$ & 46.9 & $2.7-802.6$ & 0.0079 * \\
\hline \multirow{2}{*}{ Region } & Gyeongbuk & 361 & 0 & 0.1 & $0-0.9$ & 0.0451 * \\
\hline & Gyeongnam & 376 & $9(2.4)$ & 18.3 & $1.1-312.3$ & 0.0451 * \\
\hline \multirow{3}{*}{ Season } & Spring & 282 & 0 & 0.1 & $0-1.5$ & 0.0886 \\
\hline & Summer & 178 & $9(5.1)$ & 3.0 & $1.2-7.5$ & 0.0166 * \\
\hline & Autumn & 277 & 0 & 0.1 & $0-1.5$ & 0.0924 \\
\hline \multicolumn{2}{|c|}{ Total } & 737 & $9(1.2)$ & & $0.4-2.0$ & \\
\hline
\end{tabular}

${ }^{1}$ RR, relative risk; ${ }^{2} 95 \%$ CI, $95 \%$ confidence interval. ${ }^{*}$ Statistical significance was assessed by the following Bonferroni-adjusted $p$-values: Sex $(<0.025)$, age $(<0.0167)$, breed $(<0.025)$, region $(<0.025)$, and season $(<0.0167)$. 


\subsection{Molecular Analyses}

Phylogenetic analysis revealed that the T. orientalis $18 \mathrm{~S}$ rRNA (Figure 1) and MPSP (Figure 2) nucleotide sequences obtained in this study were clustered with the previously deposited T. orientalis sequences in GenBank, as shown in Figures 1 and 2.

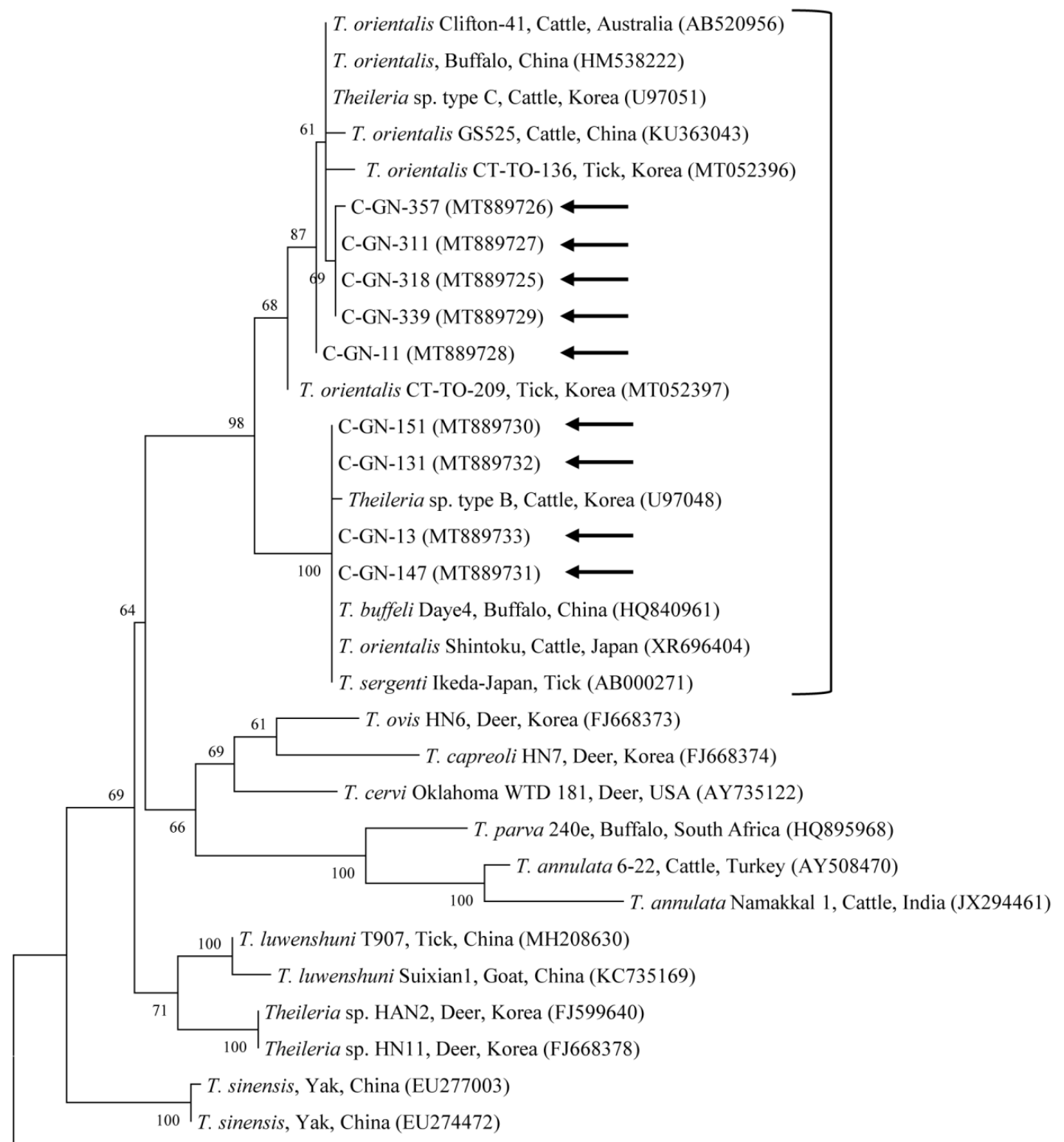

Babesia orientalis DaYe, China (HQ840969)

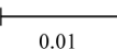

Figure 1. Phylogeny of Theileria spp. on the basis of $18 \mathrm{~S}$ rRNA nucleotide sequences created using the maximum-likelihood method. Black arrows denote the sequences reported in the current study. The GenBank accession numbers of the other sequences are shown in parentheses. Babesia orientalis is used as the outgroup. Bootstrap support levels (1000 replicates) are shown by branch numbers and the number of substitutions for each nucleotide is indicated by the scale bar. 


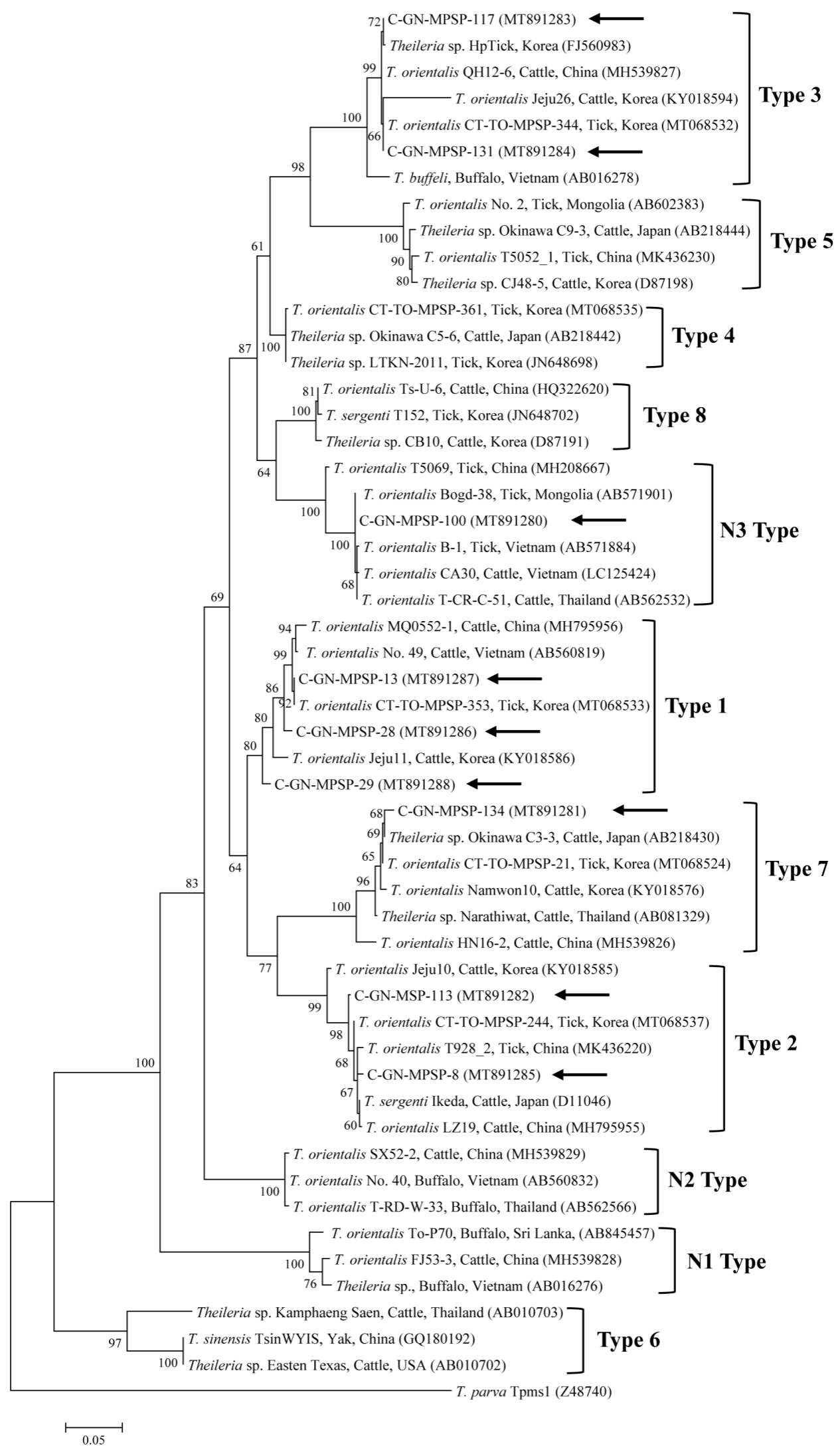

Figure 2. Phylogeny of Theileria spp. on the basis of MPSP gene sequences constructed using the maximum-likelihood method. Black arrows denote the sequences described in the current study. The GenBank accession numbers of all other sequences are given in parentheses. Theileria parva is used as the outgroup. Bootstrap support levels (1000 replicates) are shown by branch numbers and the number of substitutions for each nucleotide is indicated by the scale bar. 
The nine T. orientalis $18 \mathrm{~S}$ rRNA sequences detected in this study had $97.7-99.9 \%$ identity with each other. They also shared $97.5-99.9 \%$ identity with those of previously reported T. orientalis $18 \mathrm{~S}$ rRNA sequences in GenBank. The T. orientalis MPSP nucleotide sequences were categorized into five genotypes: Types 1, 2, 3, 7, and N3. Among the nine sequences, three, two, two, one, and one isolates were assigned to types $1,2,3,7$, and N3, respectively. The three, two, and two MPSP sequences of types 1,2 , and 3 detected in this study had $97.5-98.4 \%, 98.8 \%$, and $99.6 \%$ identity with each other, respectively. In addition, they shared $98.5-99.8 \%, 99.0-99.5 \%$, and $98.1-100 \%$ identity with the T. orientalis MPSP nucleotide sequences previously reported in GenBank, respectively. The single sequences of types 7 and N3 that we found each shared $98.2-98.9 \%$ and $98.6-99.3 \%$ identity with those T. orientalis MPSP nucleotide sequences previously deposited in GenBank, respectively. The representative sequences reported in the current study were deposited to GenBank, with the following accession numbers: MT889725-MT889733 (Theileria spp. 18S rRNA) and MT891280-MT891288 (Theileria spp. MPSP).

\section{Discussion}

Theileriosis is one of the most significant hemoprotozoan diseases infecting domestic animals, most commonly cattle and sheep, in tropical and subtropical regions and results in significant economic loss [3].

Recently, T. orientalis was detected in samples from South Korea using the $18 \mathrm{~S}$ rRNA gene in ticks $(3.7 \%, 21 / 566$ pools) infesting cattle in 2010-2011 [14], in H. longicornis ticks $(5.0 \%, 29 / 576)$ from cattle in 2014-2018 [15], in cattle $(21.7 \%, 96 / 443)$ in 2009 [11], and in cattle $(23.2 \%, 69 / 298)$ in 2014-2015 [8]. Compared with other previous studies in South Korea, we detected a relatively low prevalence of T. orientalis in cattle $(1.2 \%, 9 / 737)$. This may be due to differences in the cattle breeds, region, seasons, climate, and ticks.

The prevalence of $T$. orientalis was significantly higher in female cattle than in males. Males are mainly reared for beef production in South Korea; they are usually bred for less than three years. On the other hand, females are reared for reproduction and dairy production and have a longer lifetime and, subsequently, longer exposure period to ticks. In the present study, female dairy cattle had a higher T. orientalis prevalence than male dairy cattle. These results are similar to those of a previous study in South Korea, which reported that the prevalence of T. orientalis in female cattle $(23.3 \%, 94 / 403)$ was higher than that in male cattle [11].

T. orientalis was only detected in dairy cattle. The reason for this could be due to differences in cattle management and breeds. Dairy cattle are typically raised in barns near grassland regions in South Korea; therefore, they are more susceptible to contacting ticks. Furthermore, only cattle from two farms were infected with $T$. orientalis. All the infected cattle were permitted to graze near grassland regions, exposing them to ticks. Similar results were reported previously in South Korea, where the prevalence of $T$. orientalis in dairy cattle $(27.9 \%, 82 / 294)$ was higher than in other breeds [11]. In the current study, significant differences in the prevalence of bovine T. orientalis were observed with age. Older cattle had a higher T. orientalis prevalence compared with young cattle. This tendency in the age-involved prevalence was same as that in a previous cattle study [11].

T. orientalis infections were only detected in the cattle raised in the Gyeongnam Province. The Korean Peninsula is steadily shifting to a subtropical climate [16]. Moreover, the Gyeongnam Province is at a lower latitude in the mainland than in the Gyeongbuk Province. This biogeoclimatic difference could possibly explain the detected differences in the prevalence of TBPs and ticks. For instance, cattle were positive for T. orientalis $(96.2 \%, 25 / 26)$ from Jeju Island, which is at a lower latitude in South Korea [9]. In addition, TBPs including T. orientalis were detected in several ticks in varying proportions in different areas, and TBP infections in ticks were more abundant in the southern region than other regions in South Korea, in both the nymph and adult stages [15]. The higher prevalence of $T$. orientalis infection at the lower latitude may also be due to environmental changes, such as humidity, rainfall, and temperature, due to climate change, resulting in increased tick activity and reproduction [9]. 
Most regions of South Korea are mountainous, and summer provides the ideal conditions for ticks. A previous study has suggested that the prevalence of Theileria infection is more related to the season, mainly to the emergence of ticks and their activity levels [17]. A notable increase in tick infestation in the mountainous regions during summer may induce increased T. orientalis infection rates, resulting in notable changes in red blood cell profiles after grazing [17]. This study also presented significantly high prevalence of $T$. orientalis in summer.

The MPSP gene is conserved among different geographic isolates and is expressed in the intraerythrocytic stage of T. orientalis. As a result, it is the most frequently used marker and has been used for phylogenetic and epidemiological analyses [4]. The T. orientalis MPSP gene sequence comprises at least 11 different MPSP genotypes (types 1-8 and N1-N3) in sheep, cattle, ticks, and water buffaloes [4]. Of the 11 MPSP genotypes, ikeda (type 1), chitose (type 2), buffeli (type 3), types 4-8, and N1-N3 have been reported globally [4]. Among them, type 6 has been detected in cattle and yaks and is identified as T. sinensis [18].

Recently, genotype studies in South Korea have detected Theileria MPSP genotypes 1, 2, 4, and 8 in ticks $(2.7 \%, 15 / 556)$ from grazing cattle [19]; types $1-4$ and $7(5.0 \%, 29 / 576)$ in ticks from cattle [15]; types 1,2 , and $7(17.7 \%, 12 / 68)$ in cattle [10]; and types $1-3$ and $7(41.3 \%, 57 / 138)$ in cattle [9]. In China, types $1-5,7, \mathrm{~N} 3$, and novel N4 of the Theileria MPSP gene $(4.5 \%, 67 / 1488)$ were detected in ticks [20] and types $1-5,7, \mathrm{~N} 1$, and N2 of the Theileria MPSP gene $(36.5 \%, 95 / 260)$ were detected in cattle [21]. In this study, types $1-3,7$, and N3 of the Theileria MPSP gene $(1.2 \%, 9 / 737)$ were detected in cattle. To the best of our knowledge, this is the first study to describe the presence of Theileria MPSP genotype N3 in South Korea. However, among the nine MPSP genes isolated, only one sequence is genotype N3.

Of the five MPSP genotypes identified, type $1(33.3 \%, 3 / 9)$ was the most commonly identified genotype in ticks. In previous studies in South Korea, type 1 in cattle [10], type 2 in cattle [9], types 1 and 2 in ticks infesting cattle [15], and types 2 and 4 in ticks infesting cattle [19] were also prominent. These results suggest that types 1 and 2 are distributed nationwide and maybe related to a potential risk of theileriosis in South Korea.

Among the 11 genotypes, to date, only MPSP types 1 (chitose) and 2 (ikeda) have been associated with the clinical form of oriental theileriosis. Of these, type 2 is more pathogenic in cattle, causing severe anemia, parasitemia, and death [4]. As the clinical presentation of cattle was beyond the scope of the present study, further studies are warranted to identify the correlation between clinical symptoms and pathogenic strains of T. orientalis.

In addition to genetic variability, some risk factors were also analyzed in the present study. However, further studies are warranted to assess the regional distributions and epidemiological importance of genetic divergence in Theileria isolates. This information will help in developing efficient control and prevention methods for oriental theileriosis. This is the first report of the MPSP genotype N3 in South Korea, increasing our understanding of the genetic diversity of T. orientalis in domestic livestock. Although the prevalence of bovine T. orientalis infection was low, the present study provides data on the geographical distribution and prevalence of bovine theilerioses in this region.

\section{Materials and Methods}

\subsection{Ethics Statement}

This research was performed between 2018 and 2019 and did not require approval from the Institutional Animal Care and Use Committee at Kyungpook National University, which regulates laboratory animals maintained in indoor facilities only. Blood samples were collected from cattle by practicing veterinarians at local clinics, during periodic medical checkups, after verbal consent from farmers. 


\subsection{Samples}

In 2019, 3,645,190 cattle were reared on 100,175 farms in South Korea, including 750,686 (20.6\%) cattle on $20,822(20.8 \%)$ farms in Gyeongbuk Province and 362,464 (9.9\%) cattle on 13,566 (13.5\%) farms in Gyeongnam Province [22]. The sample size was decided statistically using a formula with an expected disease prevalence of $10 \%$, a confidence level of $95 \%$, and an accepted absolute error of $5 \%$ using a simple random sampling strategy [23].

In accordance with the formula, at least 138 samples were required. In this study, 737 cattle (Hanwoo, native Korean brown cattle; Holstein, dairy cattle) blood samples were collected from 41 cattle farms from the Gyeongsang region, including Gyeongbuk (GB) and Gyeongnam (GN) Provinces, between 2018 and 2019 (Figure 3). Data on age, sex, breed, region, and season were documented for each blood sample obtained.

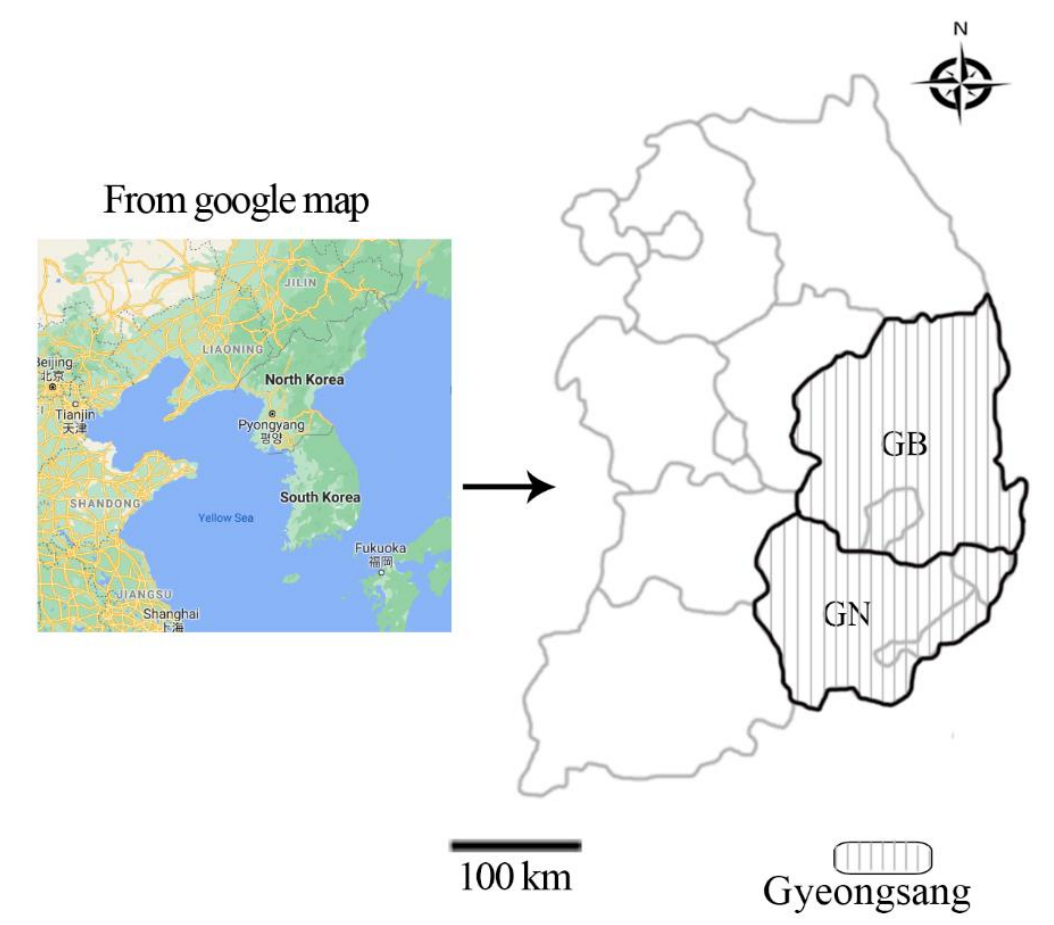

Figure 3. Map of South Korea showing the regions from which the cattle blood samples were collected for the detection of Theileria spp. from the Gyeongsang region (vertical lines), including Gyeongbuk (GB) and Gyeongnam (GN) Provinces.

\subsection{DNA Extraction and PCR}

Whole blood samples were used for genomic DNA extraction using the DNeasy Blood and Tissue Kit (Qiagen, Melbourne, Australia) following the given protocol, and the DNA quality and quantity thereof were measured with a NanoDrop ${ }^{\mathrm{TM}} 2000$ spectrophotometer (Thermo Fisher Scientific, Wilmington, DE, USA). To detect hemoprotozoan $18 \mathrm{~S}$ rRNA, blood samples were first tested for piroplasm infection via PCR using the commercial AccuPower Babesia and Theileria PCR Kit (Bioneer). Then, positive samples were retested using piroplasm $18 \mathrm{~S}$ rRNA common primers, BT-F1 (5'-GGTTGATCCTGCCAGTAGT-3') and BT-R2 (5'-TTGCGACCATACTCCCCCCA-3') primer set [16] and the MPSP genes, forward (5'-CACGCTATGTTGTCCAAGGAG-3') and reverse (5'-TGTGAGACTCAATGCGCCTA-3') primer set, of Theileria species via PCR [24]. A negative control, a sample without DNA, was included for each PCR reaction. 


\subsection{DNA Cloning}

Purification of the amplified gene fragments were done using the QIAquick Gel Extraction Kit (Qiagen) and then inserted into the pDrive vector (Promega, Madison, WI, USA) according to the given instructions. Escherichia coli DH5 $\alpha$ competent cells (Thermo Fisher Scientific) were transformed with the resulting constructs. Following bacterial incubation at $37^{\circ} \mathrm{C}$ overnight, the Plasmid Miniprep Kit (Qiagen) was used for plasmid purification based on the given instructions.

\subsection{DNA Sequencing and Phylogenetic Analysis}

Recombinant plasmids were sequenced using each 18S rRNA and MPSP gene primers by Macrogen (Seoul, South Korea), and sequences were analyzed using the multiple sequence alignment program CLUSTAL Omega (version 1.2.1, http://www.clustal.org/omega/). Results of sequence alignments were modified using BioEdit (version 7.2.5, http://www.mbio.ncsu.edu/BioEdit/bioedit.html), and phylogenetic analysis was done with MEGA (version 6.0, https://www.megasoftware.net/) using the maximum-likelihood method based on the Kimura 2-parameter distance model. The aligned sequences were evaluated with a similarity matrix. The stability of phylogenetic tree was estimated by bootstrap analysis with 1000 replicates.

\subsection{Statistical Analysis}

Statistical analysis was done using the analytical software package GraphPad Prism version 5.04 (GraphPad Software Inc., La Jolla, CA, USA). The Pearson's chi-square test was used to analyze tables with more than two variables and the Fisher's exact test was used to assess $2 \times 2$ tables. To test which cattle had an increased risk of $T$. orientalis infection within each category, each group was compared with the remaining population enrolled in the study for pairwise comparisons and $p$-values were adjusted using Bonferroni correction. A relative ratio was also calculated. Statistical significance was determined with a $p$-value of $\leq 0.05$ and a $95 \%$ CI was determined for all estimates.

Author Contributions: Conceptualization, D.K. and M.-G.S.; official analysis, M.-G.S.; funding acquisition, D.K.; methodology, D.K.; supervision, M.-G.S.; validation, M.-G.S.; Writing—original draft, D.K.; writing—editing and review, M.-G.S. All authors have read and agreed to the published version of the manuscript.

Funding: This research was funded by the Ministry of Education (Grant No. NRF-2016R1D1A1B02015366).

Acknowledgments: This research was supported by a grant from the Basic Science Research Program through the National Research Foundation of South Korea (NRF).

Conflicts of Interest: The authors declare no conflict of interest.

\section{References}

1. Vayssier-Taussat, M.; Cosson, J.F.; Degeilh, B.; Eloit, M.; Fontanet, A.; Moutailler, S.; Raoult, D.; Sellal, E.; Ungeheuer, M.N.; Zylbermann, P. How a multidisciplinary 'One Health' approach can combat the tick-borne pathogen threat in Europe. Future Microbiol. 2015, 10, 809-818. [CrossRef]

2. Schnittger, L.; Rodriguez, A.E.; Florin-Christensen, M.; Morrison, D.A. Babesia: A world emerging. Infect. Genet. Evol. 2012, 12, 1788-1809. [CrossRef] [PubMed]

3. Uilenberg, G. International collaborative research: Significance of tick-borne hemoparasitic diseases to world animal health. Vet. Parasitol. 1995, 57, 19-41. [CrossRef]

4. Sivakumar, T.; Hayashida, K.; Sugimoto, C.; Yokoyama, N. Evolution and genetic diversity of Theileria. Infect. Genet. Evol. 2014, 27, 250-263. [CrossRef] [PubMed]

5. Aktas, M.; Altay, K.; Dumanli, N. A molecular survey of bovine Theileria parasites among apparently healthy cattle and with a note on the distribution of ticks in eastern Turkey. Vet. Parasitol. 2006, 138, 179-185. [CrossRef]

6. Kamau, J.; de Vos, A.J.; Playford, M.; Salim, B.; Kinyanjui, P.; Sugimoto, C. Emergence of new types of Theileria orientalis in Australian cattle and possible cause of theileriosis outbreaks. Parasit. Vectors 2011, 4, 22. [CrossRef] [PubMed] 
7. Jia, L.; Zhao, S.; Xie, S.; Li, H.; Wang, H.; Zhang, S. Molecular prevalence of Theileria infections in cattle in Yanbian, north-eastern China. Parasite 2020, 27, 19. [CrossRef] [PubMed]

8. Kim, S.; Yu, D.H.; Kang, S.W.; Chae, J.B.; Choi, K.S.; Kim, H.C.; Park, B.K.; Chae, J.S.; Park, J. Hematological changes associated with Theileria orientalis infection in Korean indigenous cattle. Korean J. Parasitol. 2017, 55, 481-489. [CrossRef] [PubMed]

9. Park, J.; Han, Y.J.; Han, D.G.; Chae, J.B.; Chae, J.S.; Yu, D.H.; Lee, Y.S.; Park, B.K.; Kim, H.C.; Choi, K.S. Genetic characterization of Theileria orientalis from cattle in the Republic of Korea. Parasitol. Res. 2017, 116, 449-454. [CrossRef] [PubMed]

10. Park, J.; Chae, J.B.; Kim, S.; Yu, D.H.; Kim, H.C.; Park, B.K.; Chae, J.S.; Choi, K.S. Evaluation of the efficacy of ivermectin against Theileria orientalis infection in grazing cattle. BMC. Vet. Res. 2019, 15, 297.

11. Seo, M.G.; Do, J.C.; Cho, M.H.; Seo, H.J.; Kim, J.K.; Kim, Y.H.; Park, N.C.; Kwak, D.M. Prevalence of Theileria sergenti infection in cattle of eastern areas in Gyeongbuk province by PCR. Korean J. Vet. Serv. 2011, 34, 251-258. [CrossRef]

12. Ota, N.; Mizuno, D.; Kuboki, N.; Igarashi, I.; Nakamura, Y.; Yamashina, H.; Hanzaike, T.; Fujii, K.; Onoe, S.; Hata, H.; et al. Epidemiological survey of Theileria orientalis infection in grazing cattle in the eastern part of Hokkaido, Japan. J. Vet. Med. Sci. 2009, 71, 937-944. [CrossRef] [PubMed]

13. Khukhuu, A.; Lan, D.T.; Long, P.T.; Ueno, A.; Li, Y.; Luo, Y.; Macedo, A.C.; Matsumoto, K.; Inokuma, H.; Kawazu, S.; et al. Molecular epidemiological survey of Theileria orientalis in Thua Thien Hue Province, Vietnam. J. Vet. Med. Sci. 2011, 73, 701-705. [CrossRef] [PubMed]

14. Kang, S.W.; Doan, H.T.; Choe, S.E.; Noh, J.H.; Yoo, M.S.; Reddy, K.E.; Kim, Y.H.; Kweon, C.H.; Jung, S.C.; Chang, K.Y. Molecular investigation of tick-borne pathogens in ticks from grazing cattle in Korea. Parasitol. Int. 2013, 62, 276-282. [CrossRef] [PubMed]

15. Seo, M.G.; Kwon, O.D.; Kwak, D. Genotypic Analysis of Piroplasms and Associated Pathogens from Ticks Infesting Cattle in Korea. Microorganisms 2020, 8, 728. [CrossRef] [PubMed]

16. Seo, M.G.; Yun, S.H.; Choi, S.K.; Cho, G.J.; Park, Y.S.; Cho, K.H.; Kwon, O.D.; Kwak, D. Molecular and phylogenetic analysis of equine piroplasms in the Republic of Korea. Res. Vet. Sci. 2013, 94, 579-583. [CrossRef]

17. Choi, K.S.; Yu, D.H.; Chae, J.S.; Park, B.K.; Yoo, J.G.; Park, J. Seasonal changes in hemograms and Theileria orientalis infection rates among Holstein cattle pastured in the mountains in the Republic of Korea. Prev. Vet. Med. 2016, 127, 77-83. [CrossRef]

18. Liu, A.; Guan, G.; Liu, Z.; Liu, J.; Leblanc, N.; Li, Y.; Gao, J.; Ma, M.; Niu, Q.; Ren, Q.; et al. Detecting and differentiating Theileria sergenti and Theileria sinensis in cattle and yaks by PCR based on major piroplasm surface protein (MPSP). Exp. Parasitol. 2010, 126, 476-481. [CrossRef]

19. Kang, S.W.; Nguyen, L.T.; Noh, J.H.; Reddy, K.E.; Kweon, C.H.; Choe, S.E. Phylogenetic analysis of benign Theileria species based on major piroplasm surface protein (MPSP) genes from ticks of grazing cattle in Korea. Vet. Parasitol. 2012, 189, 145-152. [CrossRef]

20. Li, L.H.; Wang, J.Z.; Zhu, D.; Li, X.S.; Lu, Y.; Yin, S.Q.; Li, S.G.; Zhang, Y.; Zhou, X.N. Detection of novel piroplasmid species and Babesia microti and Theileria orientalis genotypes in hard ticks from Tengchong County, Southwest China. Parasitol. Res. 2020, 119, 1259-1269. [CrossRef]

21. Wang, J.; Yang, J.; Liu, J.; Wang, X.; Xu, J.; Liu, A.; Li, Y.; Liu, Z.; Ren, Q.; Luo, J.; et al. Molecular detection and genetic diversity of Theileria orientalis in cattle in China. Parasitol. Res. 2018, 117, 3689-3694. [CrossRef] [PubMed]

22. Korean Statistical Information Service. Number of Farms in Households and Animals in Heads by Type. Available online: http://kosis.kr/statHtml/statHtml.do?orgId=101\&tblId=DT_1EO099\&conn_path= I2\&language $=$ en/ (accessed on 1 January 2020).

23. Thrusfield, M. Veterinary Epidemiology, 3rd ed.; Blackwell Publishing: Oxford, UK, 2005.

24. Kakuda, T.; Shiki, M.; Kubota, S.; Sugimoto, C.; Brown, W.C.; Kosum, C.; Nopporn, S.; Onuma, M. Phylogeny of benign Theileria species from cattle in Thailand, China and the U.S.A. based on the major piroplasm surface protein and small subunit ribosomal RNA genes. Int. J. Parasitol. 1998, 28, 1261-1267. [CrossRef]

(C) 2020 by the authors. Licensee MDPI, Basel, Switzerland. This article is an open access article distributed under the terms and conditions of the Creative Commons Attribution (CC BY) license (http://creativecommons.org/licenses/by/4.0/). 